\title{
BOOKS AND PUBLICATIONS
}

\author{
All interested medical physicists are encouraged to have their names added to a list of available reviewers. Please rank your \\ interest among radiation therapy, $x$-ray imaging, nuclear medicine imaging, ultrasound imaging, MR imaging, radiation \\ injury, radiation protection, and others. Make your interest known to Chester Reft, Ph.D., Books Review Editor \\ (creft@radonc.uchicago.edu). Include your name and e-mail address in the body of the response.
}

\section{Book Reviews}

Noninvasive Instrumentation and Mea-
surements in Medical Diagnosis. Robert
B. Northrop. 525 pp. CRC Press, Boca Ra-
ton, FL, 2002. ISBN: 0-8493-0961-1. ton, FL, 2002

What are the physical and physiological principles behind an ECG system? How does a spirometer work? Can you measure blood glucose levels without drawing blood? What are the roles of Raman spectroscopy and optical interferometry in medical diagnosis? These are only a few of the questions Dr. Northrop addresses in his book. Numerous systems and techniques for noninvasive diagnosis and measurements are covered in 17 chapters organized topically either around a particular physiological measurement or around the utilization of a particular form of radiation energy. The content overlap between this book and a standard medical imaging text is, however, minimal. Only Chapter 16 is devoted to medical imaging modalities, while the major chapters of the book cover subject areas outside the typical medical physics realm: visual (optical) inspection of tissues, measurements of electric potentials and magnetic fields from the body surface, tests on naturally voided body fluids, and numerous nonimaging applications of audible sound, ultrasound, and electromagnetic radiation. Measurements of pulmonary function, body temperature, blood pressure, as well as basal metabolism are also presented. Advanced concepts of current research interest such as fluorescent tests for biomolecules and DNA microarrays are introduced in the last chapter, which is devoted to some future trends in noninvasive instrumentation and measurements. The text is based on the author's 25-year experience in teaching a biomedical instrumentation course at the University of Connecticut and on his personal research on noninvasive medical instrumentation prototype systems. For instance, in a section of Chapter 14, a potential Closed-Loop Constant-Phase U1trasound prototype system for the measurement of tissue glucose level is described. The system consists of an ultrasound receiver and a transmitter attached to a subject's ear lobe and an electronic circuit that maintains a constant phase lag between the transmitter and the receiver by automatically adjusting the frequency of a voltage controlled oscillator. With this system the author proposes to detect changes in tissue glucose levels by de- tecting the variation of the speed of ultrasound due to the small increase in the density of the sound-conducting medium (ear lobe) due to elevated plasma glucose concentration.

Even though the material in some chapters is rather descriptive, medical physicists seeking to satisfy a passing curiosity will find the book unsuitable for light perusal. Conceived as a classroom text, it certainly expects a few equations to be worked out and some college electronics and physiology textbooks to be opened again. The topics are consistently, clearly presented, with plenty of excellent, detailed illustrations, numerical examples, and parameter values. The short introductions on the history of certain systems are also quite entertaining. The numerous references to commercially available systems, patents, research papers, classical sources as well as World Wide Web sites are valuable. In summary, this text will be a worthy addition to the library of medical physicists involved in teaching or research utilizing noninvasive instrumentation and measurement.

\section{Reviewed by Dimitre Hristovis}

Dimitre Hristovis is a senior medical physicist with the System Concept Group at Siemens Medical Solutions, Concord, $C A$. His primary research interest is in the area of image-guided radiation therapy.

A Practical Guide to IntensityModulated Radiation Therapy. Memorial Sloan Kettering Cancer Center. 423 pp. Medical Physics Publishing, Madison, WI, 2003. Price: \$125.00. ISBN: 1-93052413-7.

This timely book on intensitymodulated radiation therapy (IMRT) consists of 19 chapters with an insightful foreword contributed by a prominent radiation oncologist. All the chapters were written by physicists, radiation oncologists, and a radiologist from Memorial Sloan Kettering Cancer Center (MSKCC). The book covers a remarkably broad range of topics in IMRT. Each chapter has a content list and is accompanied by comprehensive references.

The first chapter discusses applications of imaging tools (MR spectroscopy and PET) to identify biological and functional states of tumor and eventually to boost the dose or "paint" a part of the CTV for improved local control. Chapter 2 is a thorough summary of the book. If one wants to know what IMRT is and how it is prac- ticed, this chapter serves as an excellent introduction. Chapters 3 and 4 present algorithms used for IMRT calculations. Chapter 3 gives a detailed description of the inverse planning algorithm using the conjugate gradient optimization methods and a quadratic objective function. In Chapter 4 the authors discuss a method to generate leaf motion data for the sliding window technique. A dose calculation method, which is routinely used at MSKCC for treatment plan evaluation and has replaced patient-specific dose measurements using ionization chambers and films, is given in Chapter 5. This chapter is an invaluable resource for many users of future commercial software. The computational algorithm has not been described in the open literature in such an exhaustive manner. Chapter 6 discusses step-by-step procedures of the IMRT treatment planning process. The discussion is focused on the MSKCC system, but the overall procedure may apply to IMRT using any other system. In Chapters 7 and 8 the author gives a very detailed and well-written discussion on acceptance testing, commissioning, and routine QA procedures. However, neither these chapters nor any other section in the book discuss the commissioning procedures of treatment planning software. Chapters 9 to 14 are devoted to site-specific descriptions of clinical implementation of IMRT. The disease sites covered are prostate, head/neck, pediatric cancers, breast, lung, and sites requiring large fields such as endometrial cancers. All these chapters were co-authored by medical physicists and radiation oncologists. Unique features of some of these chapters are inclusions of site-specific optimization templates with standard beam arrangements and constraints imposed on targets and critical structures. Chapter 15 provides an up-to-date review of applications of NTCP and TCP models to IMRT treatment planning optimization. The discussion is a very good introduction to the subject, which is becoming more important as more clinical data become available. Chapter 16 presents MSKCC data and analyses of patient setup uncertainties and EPID applications on verification of delivered dose and fluence distributions. In Chapter 17 the authors discuss techniques for minimizing the geometric uncertainty due to breathing using both the deep inspiration breath hold method and the Varian Real-Time Position Management system. This chapter also covers techniques to monitor and evaluate intra- and inter-fraction motion of patients. 
Chapter 18 demonstrates the usefulness of a stereotactic body immobilization technique to treat recurrent paraspinal tumors. The final chapter, Chapter 19, is a short but very informative discussion on accelerator room shielding issues as related to IMRT.

The book entitled as "Practical" is truly a practical manual for users and potential users of IMRT delivery systems similar to the MSKCC system. The book covers all technical aspects essential to high quality treatment with this new modality. The reviewer hopes to see more books like this focusing on other commercially available IMRT systems. The book is a handy technical resource for medical physicists initiating an IMRT program as well as for experienced IMRT users. The book is also recommended for radiation oncologists who are particularly interested in the technological aspects of IMRT.

Reviewed by Yoichi Watanabe

Yoichi Watanabe, Ph.D., received postdoctoral medical physics training at MSKCC and he is currently an assistant professor of clinical radiation oncology at Columbia University. His interests are mainly in dosimetric aspects of radiation therapy physics.

Magnetic Resonance Procedures: Health Effects and Safety. Edited by Frank G. Shellock. 453 pp. CRC Press, Boca Raton, FL, 2001. Price: $\$ 89.95$ (hardcover). ISBN: 0-8493-0874-7.

As a clinical diagnostic medical physicist, one looks for reference materials that not only educate, but also prove handy enough to use routinely. This is especially true when the imaging modality is one for which you are the site "expert," but sometimes feel less than that. With a recognized name in MRI safety as both a contributor and editor, one anticipates that Magnetic Resonance Procedures: Health Effects and Safety could be that functional reference. The sixteen chapters and three appendices in the book address roughly a dozen MRI safety issues. Some of these topics would make every medical physicist's short list of MRI safety concerns: static magnetic fields (Chapter 1), gradient fields (Chapter 2), rf heating and specific absorption rate (Chapters 3, 4, and 5) and MRI during pregnancy (Chapter 8). However, the short list does not fully engage the topic of MRI safety. To round out the discussion, this volume adds discussions on acoustic noise (Chapter 6), siting and shielding a scanner (Chapter 7), patient claustrophobia and anxiety (Chapter 10), patient monitoring in a magnetic environment (Chapter 11), the use of contrast agents (Chapter 12), implants and MRI compatibility (Chapters 14, 15, Appendix I), and induced electric fields (Chapter 16). Finally, a discussion of the history and role of the FDA in MRI safety (Chapter 9); the importance of and a protocol for patient screening (Chapter 13 ); a summary of safety studies (Appen- dix II); and reference to an MRI safety website (Appendix III) are all provided.

Every chapter speaks to its subject by providing a general discussion of the theory involved and then its application to the clinical environment. Each chapter can stand on its own allowing a reader to examine a given topic with great efficiency. The table of contents also provides assistance. In addition, each chapter supports the others by providing an "in-tome" reference. For example, if one needs to address safety of magnetic resonance imaging during pregnancy one turns to the appropriate chapter. There, you can take the general view of the topic or in the table of contents for that chapter; you can focus on a particular aspect of the subject, e.g., fetal effects of contrast or rf heating. Information presented from basic research and animal studies leads to clinical applications and specific guidelines for the pregnant patient and the pregnant healthcare worker. If detail is required, one can refer to the associated chapter (e.g., contrast or rf heating) contained within the book and review the theory and application presented there. Alternatively, you can search out one of the many current references listed at the end of each chapter.

The information contained in this tome is thorough and clearly presented. Its design permits easy access to the material allowing the reader to spend the appropriate amount of time answering the MR safety question at hand. However, as with any printed material in a rapidly advancing field, the reader needs to be cautious of information obsolescence. The chapter dealing with implant compatibility, for example, will be dated quickly as new devices are introduced and some of the magnetic field strengths or gradient fields discussed may seem tame when viewed from the future. Until then, this book should prove to be that handy reference.

Reviewed by Brian M. Methé

Brian M. Methé is a diagnostic medical physicist, health physicist, and serves as radiation safety officer for a large community hospital in upstate New York. Prior to his 13 years in medical facilities, he was employed in the commercial nuclear power industry and in industrial research and development.

Therapeutic Applications of Monte Carlo Calculations in Nuclear Medicine. Edited by H. Zaidi and G. Sgouros. 364 pp. IOP, Bristol, 2003. Price: $\$ 100.00$.

Therapeutic Applications of Monte Carlo Calculations in Nuclear Medicine is a valuable collection of articles written by leaders in the field of nuclear medicine. A more appropriate title for the book may be "Therapeutic Nuclear Medicine and Applications of Monte Carlo Calculations" since a substantial fraction of the book $(\sim 1 / 2)$ is devoted to nuclear medicine, rather than the more specific topic of Monte Carlo calculations applied to nuclear medicine. The broad nature of this field necessitates a significant amount of background material, and the editors do a good job of choosing pertinent topics for the beginning chapters, such as imaging (3), and mathematical anatomy models (5) before addressing more advanced topics toward the end (e.g., MC methods and mathematical models of the skeletal system (13)). The book is thorough containing about 350 pages and 16 chapters.

The preface describes how the different chapters are related, and how topics are covered. However, it does not address the concerns of the intended audience for the book. The stand-alone nature of the individual chapters facilitates use as a reference, although one could also envision using the book as a text for a seminar/lecture series. There are a large number of different abbreviations and acronyms, and a glossary that defines these would be helpful, particularly if the book is primarily intended as a reference. Chapter 1 begins with an interesting review of the history of the MC methods followed by a discussion of the differences between electron and photon transport. The next chapter explains MC techniques used in nuclear medicine. Radiolabeled monoclonal antibodies used in radiotherapy are mentioned as a reason for the rekindled interest in MC dose calculations. As is stressed in the preface, imaging is of central importance to nuclear medicine. Chapter 3 is an overview of imaging techniques, and how these can be used to determine activity distributions. SPECT and PET imaging in particular, are compared and contrasted. Chapter 4 explains computational methods used for internal radiation dosimetry. Emphasized is that, unlike external beam treatments, it is generally not practical to experimentally measure internal doses in phantom in nuclear medicine; they must be calculated. Chapter 5 reviews different mathematical models of human anatomy, and the importance of MC calculations. Chapter 6 compares different MC codes used in nuclear medicine. Identified is EGS as the de facto standard, as well as GEANT, MCNP, and others. Also discussed are limitations of nuclear medicine dose calculations, such as the lack of predictive power associated with some of the standard phantoms. Chapter 7 describes dose point kernels, and how different MC codes are used to generate them. Chapter 8 reviews the radiobiological aspects of cancer therapy. Discussed are different tissue/organ tolerances as well as cell division delay, cell cycle redistribution, cell repair, and the oxygen effect. Also reviewed are criteria for selection of individual radionuclides. It is pointed out that almost all of nuclear medicine currently concentrates on beta emitters. Biological half-life is differentiated from the radiological half-life. Chap- 
ter 9 reviews microdosimetry of alpha emitters and Auger electron emitters. Chapters 10 and 11 describe the origins and features of the codes MABDOSE and 3D-ID used for patient specific dose calculations. Chapter 12 describes the validation of dose calculation procedures and how imaging is involved. The importance of the registration procedure of anatomical and physiological images is discussed. The simulation of imaging systems is described, emphasizing the limitations in real systems such as low count rate and pulse pile up. The computationally intensive modeling of the collimator in particular is discussed. Different radionuclides are discussed, such as the most widely used one in diagnostic imaging, ${ }^{99 \mathrm{~m}} \mathrm{Tc}$. Of interest is how MC methods can be used to model image quality degradation. The fact that ${ }^{99 \mathrm{~m}} \mathrm{Tc}$ has a simple decay scheme with a high intensity $140 \mathrm{keV}$ photon is pointed out as a desirable characteristic. Also emphasized in this chapter is that in contrast to experimental data, MC simulations allow one to assess individually the contributions of penetration and scatter. Chapter 13 is exclusively devoted to MC methods and mathematical models related to the skeletal systems. Since the bone marrow within the skeletal system is often the dose limiting "organ" in nuclear medicine, this chapter is of primary importance. Chapter 14 addressed MC methods applied to intravascular brachytherapy (IVBT) and chapter 15 discusses boron neutron capture synovectomy (BNCS) as a treatment for rheumatoid arthritis.

Given the nature of the book, i.e., many authors from different subfields writing individual chapters, it is understandable that there is a degree of format nonuniformity between chapters. However, it would be desirable if all the chapters contained a summary at the end, rather than only a few. Nevertheless, this is a very useful compilation. The information is presented in a clear and accessible fashion and is very well documented. This book will find widespread use as a reference and/or a text for those with some experience in the field, wanting to learn more, in this important and growing field of research and clinical practice.

Reviewed by Lars Ewell

Lars Ewell is a physics resident in the Department of Radiation Oncology at the University of Michigan. Among his research interests are dosimetry and Monte Carlo simulations. Prior to coming to the University of Michigan, he was a research associate at Brookhaven National Laboratory. 